
Annals of
Nutrition and
Metabolism

\section{Review Article}

Ann Nutr Metab 2021;77(suppl 3):11-19

DOI: $10.1159 / 000518498$
Received: April 20, 2021

Accepted: July 8, 2021

Published online: August 30, 2021

\title{
Impact of Delivery Mode on Infant Gut Microbiota
}

\section{Katri Korpela}

Human Microbiome Research Program, Faculty of Medicine, University of Helsinki, Helsinki, Finland

\section{Key Messages}

- Gut microbiota development is part of the physiological development of infants and should be considered in clinical practice.

- A large proportion of infants are exposed to C-section birth or intrapartum antibiotics that eliminate normal mother-toinfant transmission of beneficial microbes, altering the development of their gut microbiota and consequently immune and metabolic development.

- Breastfeeding and probiotics are especially important for infants with disrupted gut microbiota colonization.

- Faecal microbiota transplant from mother to neonate has been shown to restore normal gut microbiota in C-sectionborn infants but requires careful screening of the mother.

\section{Keywords}

Antibiotics · Bifidobacteria - Breastfeeding - C-section . Infancy and Childhood · Microbiota - Pediatrics · Probiotics

\begin{abstract}
Microbial colonization of the neonate is an important feature of normal birth. The gut microbiota has a central role in the programming of the host's metabolism and immune function, with both immediate and long-term health consequences. During vaginal birth, the infant is exposed to diverse maternal microbes, of which specific faecal microbes colonize the infant's gut. C-section eliminates the infant's contact
\end{abstract}

with maternal microbes, preventing vertical transmission of gut microbes. Consequently, infants are colonized by bacteria from the environment, including potential pathogens from the hospital environment. Recent studies have shown that intrapartum antibiotic exposure has a C-section-like effect on the infant gut microbiota. While the composition of the gut microbiota largely normalizes during the first year of life, epidemiological studies suggest that the aberrant early microbial exposures have long-term immunological and metabolic consequences. Because of the high prevalence of procedures that prevent normal gut microbiota development, effective methods to normalize the gut microbiota of neonates are urgently needed. Even more importantly, attention should be paid to the microbiota imbalance in C-sectionborn and antibiotic-exposed infants in clinical practice. Breastfeeding and probiotics are particularly important for infants with disrupted gut colonization.

๑ 2021 S. Karger AG, Basel

\section{Colonization and Early Development of Infant Gut Microbiota}

Although the notion of infants being born sterile has been contested by the detection of both bacteria and bacterial DNA in the placenta, amniotic fluid, and meconium passed within hours of birth, the expert consensus is currently that micro- 


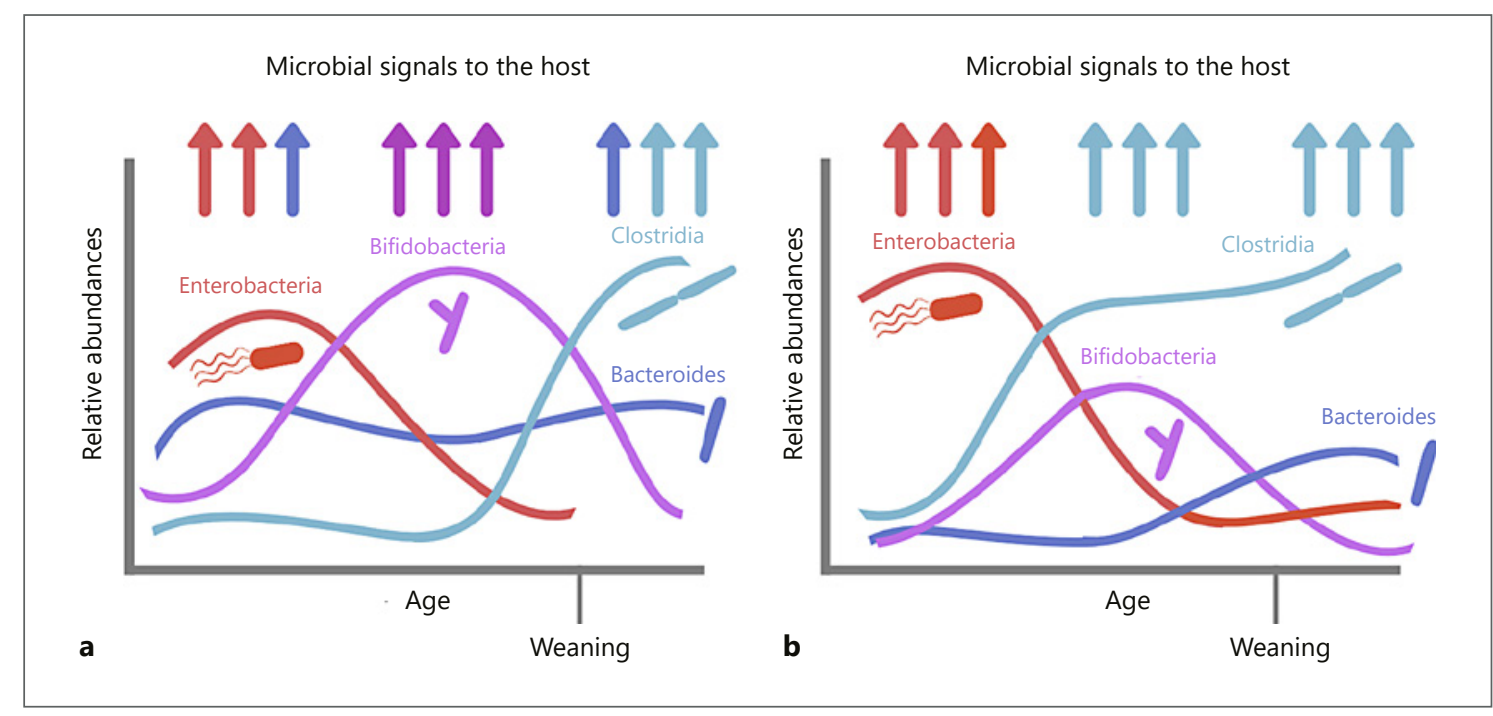

Fig. 1. Temporal development of the relative abundances of the dominant classes of bacteria in the infant gut in vaginally born, breastfed infants (a) and CS-born infants (b). CS, C-section.

bial colonization of the infant, that is, the establishment of replicating microbial cells, does not commonly occur in healthy pregnancies [1, 2]. In any case, all infants are colonized by microbes during birth. The microbes that have been observed in utero do not include the normal infant gut microbes, which must be acquired during birth.

During birth, infants are exposed to maternal vaginal, faecal, and skin bacteria, which colonize the infant body sites that provide a suitable habitat and nutrients. Each bacterial species has specific habitat requirements and cannot permanently colonize inhospitable body sites. The infant gut is colonized at birth by maternal gut microbes [3-8], while maternal vaginal microbes do not colonize the infant gut $[7,9,10]$. Not all maternal gut bacteria have the ability to colonize infants, and it is important to note that the gut microbiota is not transmitted as a whole community, but only specific members are able to establish persistent populations. The dominant maternally derived colonizers of the infant gut are bifidobacteria and Bacteroides [6, 7]. Several species of Bifidobacterium and Bacteroides have the capacity to ferment human milk oligosaccharides (HMOs) [11], which very likely explains their success in colonizing the infant gut [5]. While certain infant gutadapted Bifidobacterium strains, such as B. longum subsp. infantis, B. bifidum, and $B$. breve, are specialized in $\mathrm{HMO}$ utilization [12], Bacteroides spp. are substrate generalists. Bacteroides fragilis, thetaiotaomicron, and vulgatus are efficient HMO utilizers [11]. They exploit the same pathways for host mucus and $\mathrm{HMO}$ degradation and are able to degrade a broad range of HMOs [13]. In contrast, Firmicutes and Proteobacteria generally do not degrade HMOs, with the exception of certain Lactobacillus, Streptococcus, and Enterococcus strains with narrow HMO utilization spectra [11].

The importance of early maternal colonization is exemplified by the finding that maternally derived microbes colonize the infant essentially permanently, while non-maternal microbes are more transient [6]. This is suggestive of special compatibility between infant and maternal microbes, potentially related to partly genetically determined factors such as breast milk oligosaccharide composition, gut mucus composition, and immune system.

While the gut microbiota is generally characterized by tremendous interindividual variation, the microbiota development during infancy follows a fairly clear and uniform pattern, dependent on birth mode (Fig. 1). During the first year of life, ecological succession takes place in the infant gut with a consistent temporal pattern across cultures [14]. The first stools harbour a highly diverse microbiota of mostly transient passers-by [7]. After the first few days, the diversity decreases as specific microbes bloom while others disappear [7]. Initially dominant species include aerotolerant taxa such as staphylococci, enterococci, and streptococci as well as enterobacteria $[12,14]$. Within weeks, the composition changes as breast milk oligosaccharide-degrading bifidobacteria outgrow. Bifidobacteria are usually the dominant group until weaning after which taxa that utilize plant-based carbohydrates, mainly members of the clostridial families Lachnospiraceae and Ru-
Korpela 
Table 1. Consistent gut microbiota patterns observed in CS-born infants compared to vaginally born

\begin{tabular}{llll}
\hline & Increased in CS & Decreased in CS & $\begin{array}{l}\text { Reference (large birth cohort } \\
\text { studies or meta-analyses) }\end{array}$ \\
\hline $\begin{array}{l}\text { Bacteroides } \\
\text { Bifidobacteria }\end{array}$ & $x$ & $\times$ & {$[12,14,16,17]$} \\
Clostridia & $x$ & & {$[12,14,16]$} \\
Gram-negative pathogens & $x$ & & {$[12]$} \\
Gram-positive pathogens & $x$ & {$[14,18]$} \\
\hline
\end{tabular}

minococcaceae, begin to gradually increase in relative abundance [14]. The abundance of Bacteroides can be high already very early and remains fairly stable through the early development [14]. This likely reflects the generalist nature of Bacteroides species in terms of substrate utilization potential, including utilization of both diverse dietary polysaccharides and host-derived glycans [13]. There are some geographical differences in the average gut microbiota composition of infants. African infants show an increased relative abundance of Bacilli, while North American infants have more Bacteroidetes and less Actinobacteria than infants in other continents [14]. Whether these differences are caused by technical or biological reasons is unknown.

\section{Impact of C-Section Birth on Gut Microbiota}

The natural microbiota transmission from mother to infant is disrupted by $\mathrm{C}$-section (CS) birth [6], resulting in a deviation of microbiota development, most notably in the first 6-12 months of life [14]. In fact, CS birth is perhaps the factor with the most uniform effects on the gut microbiota across individuals and studies. A large study on nearly 600 infants found delivery mode to be the most important factor influencing infant gut microbiota composition, with a stronger effect than postnatal antibiotic treatments [15]. A particularly prominent and ubiquitous feature of the CS-born infant's gut microbiota is the low relative abundance of bifidobacteria and almost total lack of Bacteroides [14], that is, the taxa that would normally be obtained from the mother at birth and that are largely responsible for breast milk oligosaccharide fermentation in the infant gut. These patterns are observed in practically all studies investigating the impact of CS on infant gut microbiota (Table 1), including several large cohort studies of $600-$ 1,000 infants each [15-18]. In addition, the relative abundance of pathogenic bacteria is often increased in CS-born infants. These taxa are common in the hospital and are transmitted from hospital surfaces to new-born infants [19].
It has been suggested that in terms of microbial contact, CS delivery after rupture of membranes would more closely resemble vaginal delivery than elective CS in which the infant has no contact with maternal birth canal microbiota. While the exposure of mother and infant to the labour process undoubtedly has physiological effects, there is no reason to expect much benefit for the microbial colonization from labour, even with rupture of membranes, as maternal faecal microbes are unlikely to be present in the birth canal of healthy mothers. Indeed, the hypothesis has not received much support in the scientific literature. Only a few studies have compared the effect of different CS types on infant gut microbiota. Emergency CS seems to result in more drastic and long-lasting changes in infant gut microbiota $[18,20]$, possibly due to health differences. To conclude, there is currently no evidence suggesting a beneficial effect of rupture of membranes or CS during labour versus pre-labour CS on infant gut microbiota.

Thus far, only a few studies have looked into the viral component of the gut microbiota, the so-called gut virome, in early infancy. Recent results show that most of the viruses in the neonate gut are phages, that is, virus-infecting bacteria [21]. There is evidence that phages are transmitted from the mother at birth together with their host bacteria [5, 22]. Consequently, total virus and phage diversity has been shown to be reduced in CS-born infants at the age of 1 year, with particularly low abundances of Anelloviridae and Caudovirales in CS-born infants [23]. In fact, the effect of birth mode on the gut virome was much more prominent at 1 year than the effect on the bacteriome [23]. This indicates that birth is crucial for the acquisition of the gut phageome, and the potential for postnatal colonization, for example, via breast milk or the environment, is limited.

A meta-analysis of the dominant bacterial classes revealed that the differences between birth modes gradually even out and mostly disappear by the age of 12 months [14]. However, only a few studies have investigated the associations between birth mode and gut microbiota development after infancy. Reduced diversity of Bacteroides spp. has been reported up 
to the age of 2 years [24]. Another study found reduced relative abundances of several members of Lachnospiraceae and Ruminococcaceae in CS-born children at the age of 4 years [25]. Comparably, a study using fluorescent in situ hybridization found a reduced abundance of clostridia in CS-born compared to vaginally born 7 year olds [26]. A study using quantitative PCR found significant differences in gut microbiota composition between CS-born and vaginally born young adults [27]. In particular, relatives of Bacteroides fragilis and Lactobacillus sakei were reduced in CS-born children. The results suggest that birth mode effects on the gut microbiota may in some cases persist even into adulthood. Notably, the latter 2 studies employed quantitative measures (FISH and qPCR, respectively) that can estimate the absolute abundance of the bacteria, in contrast to sequencing which only reveals relative abundances. Clearly, future studies need to employ methods that allow the absolute abundances to be estimated [28].

\section{Impact of Intrapartum Antibiotics on Infant Gut Microbiota}

In most hospitals, antibiotic treatment given to the mother is an integral part of CS delivery. Therefore, there has been some unclarity as to whether the effect of CS birth is exclusively due to the lack of exposure to maternal faecal microbes or partly due to the antibiotic treatment. Considering that regardless of maternal microbiota composition, CS eliminates the contact between maternal faecal microbes and infant, thus preventing colonization, and any additional microbiota-affecting treatments seem trivial. If a microbe has no contact with the infant, it cannot colonize the infant regardless of exposure to antibiotics. This view was recently confirmed in a study comparing the effects of pre- versus post-CS antibiotic prophylaxis on the infants' gut microbiota composition [29]. No significant differences in gut microbiota composition were found between infants exposed and not exposed to maternal antibiotic prophylaxis, demonstrating that antibiotic exposure is not a driver of the CS-induced microbiota imbalance. Along the same lines, in an analysis of 700 infants, stratification by intrapartum antibiotic exposure did not alter the results regarding the effect of CS birth on infant gut microbiota [18].

The effect of antibiotic treatment during vaginal birth is an important issue in itself, since a large proportion of infants are exposed to intrapartum antibiotics either due to prophylaxis or treatment of maternal infection. In some countries, all mothers who carry group B Streptococcus are given antibiotic prophylaxis during labour. The aim of intrapartum antibiotic prophylaxis is to prevent transmission of pathogenic bac-
Table 2. Consistent gut microbiota patterns observed in vaginally born infants exposed to intrapartum antibiotics compared to those not exposed to antibiotics

\begin{tabular}{llll}
\hline & $\begin{array}{c}\text { Increased } \\
\text { in exposed }\end{array}$ & $\begin{array}{c}\text { Decreased } \\
\text { in exposed }\end{array}$ & Reference \\
\hline Bifidobacteria & & $x$ & {$[30-34]$} \\
Clostridia & $x$ & & {$[20,30,32]$} \\
Enterobacteria & $x$ & {$[20,30,32]$} \\
Streptococci & $x$ & {$[30,32]$} \\
Gram-negative pathogens & $x$ & {$[18]$} \\
Gram-positive pathogens & $x$ & {$[18]$} \\
\hline
\end{tabular}

teria from mother to infant. Antibiotics are not selective between pathogens and non-pathogens, so if the transmission of pathogens is prevented, so must be the transmission of non-pathogenic bacteria with a similar antibiotic sensitivity profile.

In studies investigating the impact of intrapartum antibiotics in vaginally born infants, bifidobacteria appear to be particularly strongly negatively affected by the antibiotic exposure (Table 2). There is discrepancy regarding the impact of intrapartum antibiotic exposure on Bacteroides, with effects varying from negative $[17,20,30]$ to neutral $[20,31-33]$ to positive [30]. Importantly, the impact of antibiotic exposure is modified by feeding practice, with formula-fed infants more severely affected [34-36]. Overall, the emerging evidence suggests that antibiotic exposure during birth can be likened to a chemical CS from the microbial perspective, eliminating the natural transmission of gut microbiota, especially bifidobacteria, from mother to infant.

\section{Consequences of Disrupted Vertical Microbial Transfer}

Due to the age-associated microbiota succession, the host receives specific microbial signals at specific age windows (Fig. 1). If these signals are altered or missing due to abnormal microbiota development, the host's immune and metabolic development may be affected. Deviations from normal microbiota succession in infants and young children usually occur without overt symptoms, but can increase the susceptibility to later health problems, such as allergic disease [37-39], autoimmune diseases [40], and overweight [41].

Bifidobacteria normally form the dominant taxon in healthy vaginally born breastfed infants, representing up to $90 \%$ of the total gut microbiota. Due to their abundance and metabolic capacities, they have important effects on gut microbiota
Korpela 
Fig. 2. Effects of low abundance of bifidobacteria in infants.

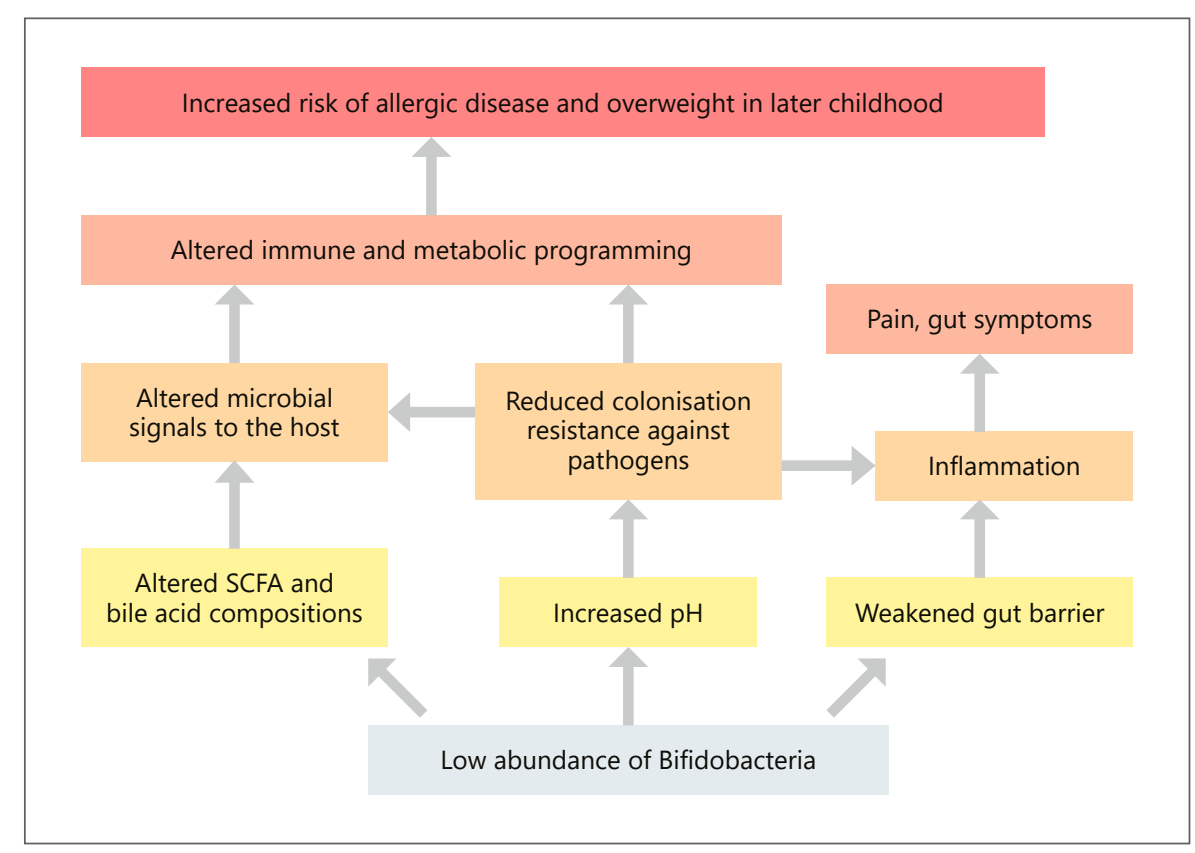

composition and function and host health (Fig. 2). Unfortunately, bifidobacteria are perhaps the most vulnerable bacterial group in infants, affected negatively by all unnatural practices such as CS birth, antibiotics, formula feeding, early weaning, and even maternal stress. Bifidobacteria are the dominant $\mathrm{HMO}$-utilizing group in the infant gut.

As a consequence of the reduced abundance of dominant $\mathrm{HMO}$ fermenters and lower fermentation activity, an increased intestinal $\mathrm{pH}$ and decreased levels of the short-chain fatty acid propionate has been observed in CS-born infants [42]. As HMOs constitute approximately $20 \%$ of all carbohydrates in breast milk [43], and the majority of them are degraded by gut bacteria into short-chain fatty acids [44], HMO fermentation represents a significant energy source for the infant. This indicates that CS-born infants do not receive the full nutritional value of breast milk. Furthermore, the altered gut $\mathrm{pH}$ has been shown to be permissive for pathogen colonization [42], and indeed several studies have observed increased abundances of pathogens in CS-born infants (Table 1). Bifidobacteria have anti-streptococcal activity, indicating that a low abundance of bifidobacteria in the gut may increase the growth of pathogenic streptococci [31]. In addition to the increase in pathogenic microbes, there is evidence that a microbiota with reduced capacity for oligosaccharide utilization degrades the intestinal mucus, potentially leading to weakened intestinal barrier [45]. This together with the increase in pathogenic microbes is likely to stimulate inflammation both systemically and locally. Indeed, signs of pain and distress in infants are correlated with low abundance of bifidobacteria $[46,47]$.

Impact of Delivery Mode
Not only are bifidobacteria important for early-life gut health but also have widespread immunological effects, which is particularly important during the time of immune system development. Indeed, numerous studies have shown that a low abundance of bifidobacteria, especially $B$. longum, and a high abundance of Proteobacteria in infancy is associated with an increased risk of allergic diseases in later childhood [48-51]. Several studies have shown that low early abundance of bifidobacteria is predictive of later adiposity, as well $[41,52,53]$.

As a demonstration of the clinical consequences of the disrupted microbial colonization, several large cohort studies and meta-analyses have shown that birth by CS is associated with long-term health effects, including increased risk of chronic immune diseases and obesity [54-56], with some of the negative health associations reaching into adulthood [54]. While some associations may be caused by confounding effects, the clear alterations in gut microbiota and the importance of the gut microbiota for host physiological development support the causal attribution.

\section{Correction of Infant Microbiota after Disrupted Colonization}

CS deliveries are increasing worldwide, affecting over $50 \%$ of infants in certain regions [57]. Additionally, over $30 \%$ of infants are exposed to antibiotics during vaginal birth in developed countries $[58,59]$. These figures are alarmingly high, suggest-

Ann Nutr Metab 2021:77(suppl 3):11-19 
Fig. 3. Studied methods of microbiota normalization in CS-born infants. CS, Csection; FMT, faecal microbiota transplantation.

\section{Benefits}

- The cheapest and least invasive method

- Promotes normal microbiota

composition and function

- Additional health benefits for both infant and mother

Breastfeeding

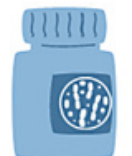

Probiotics

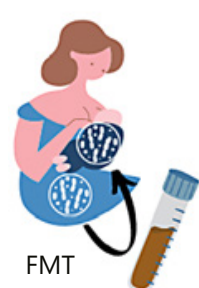

- Can be effective at normalising bifidobacterial abundance when combined with

breastfeeding or prebiotics

- Safe and widely available

- The most effective method

- Full microbiota restoration

- Restores vertical transmission of microbes from mother to infant

- One-time application
Disadvantages

- Efficacy dependent on maternal genotype

- Ineffective at full microbiota restoration
- Ineffective in formula-fed infants without added prebiotics

- Effect is strain-specific

- Ineffective at full microbiota restoration

- Requires careful screening of the mother and monitoring of the infant

- Only in hospital

- Not suitable if mother carries

potential pathogens ing that natural microbial colonization at birth is currently disrupted in up to half of infants even in regions with low CS rates and good antibiotic stewardship. Therefore, there is a pressing need to restore normal gut microbiota composition in CSborn and antibiotic-exposed infants. Only a few different methods have been studied in terms of their efficacy in restoring normal gut microbiota in CS-born infants (Fig. 3).

A study comparing the microbiota found on different infant body sites within minutes after birth and meconium samples taken within the first $24 \mathrm{~h}$ to maternal skin and vaginal samples showed, unsurprisingly, that vaginally born infants, still covered with vaginal fluids at the time of sampling, harboured vaginal microbes, and CS-born infants harboured skin microbes [60]. These results were interpreted to mean that the infant gut is colonized by maternal vaginal microbes at birth, although faecal microbiota was not sampled in the study. However, the samples were taken too early to distinguish colonizers from transient passers-by. In fact, it was shown already in 1990 that vaginal lactobacilli do not colonize the infant gut [9], and later research has confirmed this [7, 10]. The adult vagina is a very different environment compared to infant body sites, and consequently microbes adapted to the vaginal environment, predominantly specific Lactobacillus species, are unlikely to find suitable habitats on the infant body. Human vaginal lactobacilli are highly adapted to that environment and rarely observed in other body sites [61]. Therefore, vaginal microbes are not useful in terms of infant gut microbiota restoration [8]. The infant gut does harbour a low abundance of lactobacilli, but those are gut adapted or food derived, rather than of vaginal origin [61]. Whether the transient exposure to vaginal microbes during birth plays a role in, for example, immune stimulation, is currently unknown.

A few studies have tested the efficacy of probiotics as a means of microbiota restoration in CS-born infants. A 6-month daily supplementation with a probiotic product containing several Lactobacillus and Bifidobacterium strains has been shown to alleviate the CS- and antibiotic-associated microbiota disturbance in breastfed infants [62]. Importantly, the treatment reduced the incidence of allergic diseases in CS-born infants, demonstrating that gut microbiota-targeting interventions have beneficial health effects [63]. However, the effect of the probiotic intervention on the gut microbiota was dependent on breastfeeding and failed to increase bifidobacteria in the exclusively formula-fed infants [62]. In another trial, Lactobacillus reuteri-containing formula was fed to vaginally born and CS-born infants for 6 months, with beneficial effects on the abundances of bifidobacteria and lactobacilli in the CS-born group [64]. These results are very promising, demonstrating the effectiveness of such simple, safe, and cost-effective interventions. However, probiotics represent a small fraction of the total microbial diversity that normally colonize infants and cannot fully restore normal gut microbiota. Importantly, probiotics are not maternally derived. It is likely that maternal strains are adapted to the specific motherinfant dyad and have been preselected by maternal immune
Korpela 
system and gut secretions to suit her infant and her breast milk composition.

Breast milk is highly bifidogenic, so breastfeeding could be expected to restore the low abundance of bifidobacteria in CS-born infants. Exclusive breastfeeding has been found to have a beneficial impact on the gut microbiota of CS-born infants, especially regarding bifidobacteria $[65,66]$. However, the effect of breastfeeding is dependent on breast milk oligosaccharide composition [67]. The oligosaccharide 2'fucosyllactose in breast milk alleviates the effect of CS birth on the gut microbiota, but not all mothers secrete 2 'fucosyllactose [67]. The presence of fucosylated oligosaccharides in breast milk, the so-called secretor status, is determined by the mother's FUT2 genotype. The results indicate that infants of nonsecretor mothers and formula-fed infants are at a particular risk of gut dysbiosis if born by CS. Overall, the results show that breastfeeding should be considered essential for infants with disrupted gut colonization.

Due to the fact that infants are normally colonized by maternal faecal microbes during birth, birth can be seen as a faecal microbiota transplantation (FMT) event from mother to infant. In a recent pilot study, CS-born infants were given an oral FMT, a few grams of faecal matter mixed with breast milk, from their own mothers within hours after delivery [8]. The treatment fully restored normal gut microbiota composition, validating that maternal faecal microbes are the natural colonizers of the infant gut. While all procedures transferring a community of microbes from 1 person to another are inherently risky due to the potential of transferring pathogens, FMT from carefully screened healthy mothers was shown to be safe [8].

\section{References}

1 Blaser MJ, Devkota S, McCoy KD, Relman DA, Yassour M, Young VB. Lessons learned from the prenatal microbiome controversy. Microbiome. 2021;9(1):8-7.

2 Walter J, Hornef MW. A philosophical perspective on the prenatal in utero microbiome debate. Microbiome. 2021;9:1-9.

3 Makino H, Kushiro A, Ishikawa E, Kubota H, Gawad A, Sakai T, et al. Mother-to-infant transmission of intestinal bifidobacterial strains has an impact on the early development of vaginally delivered infant's microbiota. PLoS One. 2013;8:e78331.

4 Asnicar F, Manara S, Zolfo M, Truong DT, Scholz M, Armanini F, et al. Studying vertical microbiome transmission from mothers to infants by strain-level metagenomic profiling. bioRxiv. 2016. Epub ahead of print.

5 Duranti S, Lugli GA, Mancabelli L, Armanini F, Turroni F, James K, et al. Maternal inheritance of bifidobacterial communities and bifidophages in infants through vertical transmission. Microbiome. 2017; 5:66

Impact of Delivery Mode

\section{Conclusion}

Because CS eliminates the contact between infant and maternal faecal microbes, natural vertical transfer of gut microbiota from mother to infant is prevented. This results in aberrant gut microbiota composition with reduced abundance of breast milk fermenters and increased abundance of pathogens during the first year of life, possibly longer. Intrapartum antibiotic exposure during vaginal birth has a similar but somewhat weaker impact on the infant gut microbiota as CS. Accumulating evidence indicates that the disrupted gut colonization in early life carries long-term health risks, including increased risk of chronic immune diseases and overweight. Due to the high prevalence of both CS and intrapartum antibiotic use, methods to correct the infant gut microbiota are needed. Breastfeeding together with probiotics, especially those containing bifidobacteria, can be used to alleviate the microbiota imbalance and are highly recommendable for all CS-born and antibiotic-exposed infants. The efficacy of mother-to-infant FMT in correcting the infant gut microbiota has been recently demonstrated.

\section{Conflict of Interest Statement}

The writing of this article was supported by Nestlé Nutrition Institute and the author declares no other conflicts of interest.

\section{Funding Sources}

The writing of this article was supported by funding from the University of Helsinki.

6 Korpela K, Costea P, Coelho LP, Kandels-Lewis S, Willemsen G, Boomsma DI, et al. Selective maternal seeding and environment shape the human gut microbiome. Genome Res. 2018;28:561-8.

7 Ferretti P, Pasolli E, Tett A, Asnicar F, Gorfer V, Fedi S, et al. Mother-to-infant microbial transmission from different body sites shapes the developing infant gut microbiome. Cell Host Microbe. 2018;24(1):133-45.

8 Korpela K, Helve O, Kolho KL, Saisto T, Skogberg K, Dikareva E, et al. Maternal fecal microbiota transplantation in cesarean-born infants rapidly restores normal gut microbial development: a Proofof-Concept Study. Cell. 2020;183:324-34.e5.

9 Tannock GW, Fuller R, Smith SL, Hall MA. Plasmid profiling of members of the family Enterobacteriaceae, lactobacilli, and bifidobacteria to study the transmission of bacteria from mother to infant. J Clin Microbiol. 1990;28:1225-8. 
10 Sakwinska O, Foata F, Berger B, Brüssow H, Combremont S, Mercenier $\mathrm{A}$, et al. Does the maternal vaginal microbiota play a role in seeding the microbiota of neonatal gut and nose? Benef Microbes. 2017; 8:763-78.

11 Yu ZT, Chen C, Newburg DS. Utilization of major fucosylated and sialylated human milk oligosaccharides by isolated human gut microbes. Glycobiology. 2013;23:1281-92.

12 Thomson P, Medina DA, Garrido D. Human milk oligosaccharides and infant gut bifidobacteria: molecular strategies for their utilization. Food Microbiol. 2018;75:37-46.

13 Marcobal A, Barboza M, Sonnenburg ED, Pudlo N, Martens EC, Desai $P$, et al. Bacteroides in the infant gut consume milk oligosaccharides via mucus-utilization pathways. Cell Host Microbe. 2011; 10:507-14.

14 Korpela K, de Vos WM. Early life colonization of the human gut: microbes matter everywhere. Curr Opin Microbiol. 2018;44:70-8.

15 Shao Y, Forster SC, Tsaliki E, Vervier K, Strang A, Simpson N, et al. Stunted microbiota and opportunistic pathogen colonization in caesarean-section birth. Nature. 2019;574:117-21.

16 Penders J, Thijs C, Vink C, Stelma FF, Snijders B, Kummeling I, et al. Factors influencing the composition of the intestinal microbiota in early infancy. Pediatrics. 2006;118:511-21.

17 Fallani M, Young D, Scott J, Norin E, Amarri S, Adam R, et al. Intestinal microbiota of 6 -week-old infants across Europe: geographic influence beyond delivery mode, breast-feeding, and antibiotics. J Pediatr Gastroenterol Nutr. 2010;51:77-84.

18 Stokholm J, Thorsen J, Chawes BL, Schjørring S, Krogfelt KA, Bønnelykke $K$, et al. Cesarean section changes neonatal gut colonization. J Allergy Clin Immunol. 2016;138:881-9.e2.

19 Brooks B, Olm MR, Firek BA, Baker R, Thomas BC, Morowitz MJ, et al. Strain-resolved analysis of hospital rooms and infants reveals overlap between the human and room microbiome. Nat Commun. 2017; 8:1814-7.

20 Azad MB, Konya T, Persaud RR, Guttman DS, Chari RS, Field CJ, et al. Impact of maternal intrapartum antibiotics, method of birth and breastfeeding on gut microbiota during the first year of life: a prospective cohort study. BJOG. 2016;123:983-93.

21 Liang G, Zhao C, Zhang H, Mattei L, Sherrill-Mix S, Bittinger K, et al. The stepwise assembly of the neonatal virome is modulated by breastfeeding. Nature. 2020;581:470-4.

22 Siranosian BA, Tamburini FB, Sherlock G, Bhatt AS. Acquisition, transmission and strain diversity of human gut-colonizing crasslike phages. Nat Commun. 2020;11:280-11.

23 McCann A, Ryan FJ, Stockdale SR, Dalmasso M, Blake T, Ryan CA, et al. Viromes of one year old infants reveal the impact of birth mode on microbiome diversity. PeerJ. 2018;6:e4694.

24 Jakobsson HE, Abrahamsson TR, Jenmalm MC, Harris K, Quince C, Jernberg C, et al. Decreased gut microbiota diversity, delayed Bacteroidetes colonisation and reduced Th1 responses in infants delivered by caesarean section. Gut. 2014;63:559-66.

25 Fouhy F, Watkins C, Hill CJ, O'Shea CA, Nagle B, Dempsey EM, et al. Perinatal factors affect the gut microbiota up to four years after birth. Nat Commun. 2019;10:1517.
26 Salminen S, Gibson GR, McCartney AL, Isolauri E. Influence of mode of delivery on gut microbiota composition in seven year old children. Gut. 2004;53:1388-9.

27 Nagpal R, Yamashiro Y. Gut microbiota composition in healthy Japanese infants and young adults born by $\mathrm{C}$-section. Ann Nutr Metab. 2018;73 Suppl 3:4-11.

28 Jian C, Luukkonen P, Yki-Järvinen H, Salonen A, Korpela K. Quantitative PCR provides a simple and accessible method for quantitative microbiota profiling. PLoS One. 2020;15:e0227285.

29 Kamal SS, Hyldig N, Krych $Ł$, Greisen G, Krogfelt KA, Zachariassen $G$, et al. Impact of early exposure to cefuroxime on the composition of the gut microbiota in infants following cesarean delivery. J Pediatr. 2019;210:99-105.e2.

30 Stearns JC, Simioni J, Gunn E, McDonald H, Holloway AC, Thabane L, et al. Intrapartum antibiotics for GBS prophylaxis alter colonization patterns in the early infant gut microbiome of low risk infants. Sci Rep. 2017;7:16527-9.

31 Aloisio I, Mazzola G, Corvaglia LT, Tonti G, Faldella G, Biavati B, et al. Influence of intrapartum antibiotic prophylaxis against group $B$ Streptococcus on the early newborn gut composition and evaluation of the anti-Streptococcus activity of Bifidobacterium strains. Appl Microbiol Biotechnol. 2014;98:6051-60.

32 Bugmann $\mathrm{H}$. Functional types of trees in temperate and boreal forests: classification and testing. J Veg Sci. 1996;7(3):359-70.

33 Imoto N, Morita H, Amanuma F, Maruyama H, Watanabe S, Hashiguchi N. Maternal antimicrobial use at delivery has a stronger impact than mode of delivery on bifidobacterial colonization in infants: a pilot study. J Perinatol. 2018;38:1174-81.

34 Nogacka A, Salazar N, Suárez M, Milani C, Arboleya S, Solís G, et al. Impact of intrapartum antimicrobial prophylaxis upon the intestinal microbiota and the prevalence of antibiotic resistance genes in vaginally delivered full-term neonates. Microbiome. 2017:5:93.

35 Corvaglia L, Tonti G, Martini S, Aceti A, Mazzola G, Aloisio I, et al. Influence of intrapartum antibiotic prophylaxis for group B streptococcus on gut microbiota in the first month of life. $J$ Pediatr Gastroenterol Nutr. 2016;62(2):304-8.

36 Mazzola G, Murphy K, Ross RP, Di Gioia D, Biavati B, Corvaglia LT, et al. Early gut microbiota perturbations following intrapartum antibiotic prophylaxis to prevent group B streptococcal disease. PLoS One. 2016;11(6):e0157527.

37 Abrahamsson TR, Jakobsson HE, Andersson AF, Björkstén B, Engstrand $\mathrm{L}$, Jenmalm MC. Low diversity of the gut microbiota in infants with atopic eczema. J Allergy Clin Immunol. 2012;129:434.

38 Abrahamsson TR, Jakobsson HE, Andersson AF, Björkstén B, Engstrand $\mathrm{L}$, Jenmalm MC. Low gut microbiota diversity in early infancy precedes asthma at school age. Clin Exp Allergy. 2014;44: 842-50.

39 Azad MB, Konya T, Guttman DS, Field CJ, Sears MR, HayGlass KT, et al. Infant gut microbiota and food sensitization: associations in the first year of life. Clin Exp Allergy. 2015;45:632-43.

40 Kostic AD, Gevers D, Siljander H, Vatanen T, Hyötyläinen T, Hämäläinen AM, et al. The dynamics of the human infant gut microbiome in development and in progression toward type 1 diabetes. Cell Host Microbe. 2015:17:260-73.
Korpela 
41 Korpela K, Zijlmans MA, Kuitunen M, Kukkonen K, Savilahti E, SaIonen A, et al. Childhood BMI in relation to microbiota in infancy and lifetime antibiotic use. Microbiome. 2017;5:26.

42 Nagpal R, Tsuji H, Takahashi T, Nomoto K, Kawashima K, Nagata $\mathrm{S}$, et al. Gut dysbiosis following C-section instigates higher colonisation of toxigenic Clostridium perfringens in infants. Benef $\mathrm{Mi}-$ crobes. 2017:8:353-65.

43 Thurl S, Munzert M, Boehm G, Matthews C, Stahl B. Systematic review of the concentrations of oligosaccharides in human milk. Nutr Rev. 2017;75:920-33.

44 Coppa GV, Pierani P, Zampini L, Bruni S, Carloni I, Gabrielli O. Characterization of oligosaccharides in milk and feces of breastfed infants by high-performance anion-exchange chromatography. In: Anonymous bioactive components of human milk. Springer; 2001. p. 307-14.

45 Karav S, Casaburi G, Frese SA. Reduced colonic mucin degradation in breastfed infants colonized by Bifidobacterium longum subsp. infantis EVC001. FEBS Open Bio. 2018;8:1649-57.

46 Pärtty A, Kalliomäki M, Endo A, Salminen S, Isolauri E. Compositional development of Bifidobacterium and Lactobacillus microbiota is linked with crying and fussing in early infancy. PLoS One. 2012; 7:e32495.

47 de Weerth C, Fuentes S, Puylaert P, de Vos WM. Intestinal microbiota of infants with colic: development and specific signatures. Pediatrics. 2013;131:e550-8.

48 Sjogren YM, Jenmalm MC, Bottcher MF, Bjorksten B, SverremarkEkstrom E. Altered early infant gut microbiota in children developing allergy up to 5 years of age. Clin Exp Allergy. 2009;39:518-26.

49 Akay HK, Tokman HB, Hatipoglu N, Hatipoglu H, Siraneci R, Demirci $M$, et al. The relationship between bifidobacteria and allergic asthma and/or allergic dermatitis: a prospective study of $0-3$ years-old children in Turkey. Anaerobe. 2014;28:98-103.

50 Enomoto T, Sowa M, Nishimori K, Shimazu S, Yoshida A, Yamada $K$, et al. Effects of bifidobacterial supplementation to pregnant women and infants in the prevention of allergy development in infants and on fecal microbiota. Allergol Int. 2014;63:575-85.

51 Low JSY, Soh SE, Lee YK, Kwek KYC, Holbrook JD, Van der Beek EM, et al. Ratio of Klebsiella/Bifidobacterium in early life correlates with later development of paediatric allergy. Benef Microbes. 2017; 8:681-95

52 Kalliomaki M, Collado MC, Salminen S, Isolauri E. Early differences in fecal microbiota composition in children may predict overweight. Am J Clin Nutr. 2008;87:534-8.

53 Dogra S, Sakwinska O, Soh SE, Ngom-Bru C, Brück WM, Berger B, et al. Dynamics of infant gut microbiota are influenced by delivery mode and gestational duration and are associated with subsequent adiposity. mBio. 2015;6:e02419-14.

54 Hansen S, Halldorsson TI, Olsen SF, Rytter D, Bech BH, Granström $C$, et al. Birth by cesarean section in relation to adult offspring overweight and biomarkers of cardiometabolic risk. Int J Obes. 2018;42:15-9.
55 Keag OE, Norman JE, Stock SJ. Long-term risks and benefits associated with cesarean delivery for mother, baby, and subsequent pregnancies: systematic review and meta-analysis. PLoS Med. 2018;15:e1002494

56 Andersen V, Möller S, Jensen PB, Møller FT, Green A. Caesarean delivery and risk of chronic inflammatory diseases (inflammatory bowel disease, rheumatoid arthritis, coeliac disease, and diabetes mellitus): a population based registry study of 2,699,479 births in Denmark during 1973-2016. Clin Epidemiol. 2020;12:287.

57 Births by caesarean section. WHO; 2018. Available from: https:// apps.who.int/gho/data/node.main.BIRTHSBYCAESAREAN? lang=en. Accessed 2020 Sep 22.

58 Stokholm J, Schjørring S, Pedersen L, Bischoff AL, Følsgaard N, Carson CG, et al. Prevalence and predictors of antibiotic administration during pregnancy and birth. PLoS One. 2013; 8:e82932.

59 Persaud RR, Azad MB, Chari RS, Sears MR, Becker AB, Kozyrskyj AL. Perinatal antibiotic exposure of neonates in Canada and associated risk factors: a population-based study. J Matern Fetal Neonatal Med. 2015;28:1190-5.

60 Dominguez-Bello MG, Costello EK, Contreras M, Magris M, Hidalgo G, Fierer N, et al. Delivery mode shapes the acquisition and structure of the initial microbiota across multiple body habitats in newborns. Proc Natl Acad Sci U S A. 2010;107:11971-5.

61 Mendes-Soares H, Suzuki H, Hickey RJ, Forney LJ. Comparative functional genomics of Lactobacillus spp. reveals possible mechanisms for specialization of vaginal lactobacilli to their environment. J Bacteriol. 2014;196:1458-70. 7

62 Korpela K, Salonen A, Vepsäläinen O, Suomalainen M, Kolmeder $C$, Varjosalo $M$, et al. Probiotic supplementation restores normal microbiota composition and function in antibiotic-treated and in caesarean-born infants. Microbiome. 2018;6:182.

63 Kuitunen M, Kukkonen K, Juntunen-Backman K, Korpela R, Poussa $T$, Tuure T, et al. Probiotics prevent IgE-associated allergy until age 5 years in cesarean-delivered children but not in the total cohort. J Allergy Clin Immunol. 2009;123:335-41.

64 Garcia Rodenas CL, Lepage M, Ngom-Bru C, Fotiou A, Papagaroufalis $K$, Berger $B$. Effect of formula containing Lactobacillus reuteri DSM 17938 on fecal microbiota of infants born by cesareansection. J Pediatr Gastroenterol Nutr. 2016;63:681-7.

65 Yasmin F, Tun HM, Konya TB, Guttman DS, Chari RS, Field CJ, et al. Cesarean section, formula feeding, and infant antibiotic exposure: separate and combined impacts on gut microbial changes in later infancy. Front Pediatr. 2017;5:200.

66 Liu Y, Qin S, Song Y, Feng Y, Lv N, Xue Y, et al. The perturbation of infant gut microbiota caused by cesarean delivery is partially restored by exclusive breastfeeding. Front Microbiol. 2019;10:598.

67 Korpela K, Salonen A, Hickman B, Kunz C, Sprenger N, Kukkonen $\mathrm{K}$, et al. Fucosylated oligosaccharides in mother's milk alleviate the effects of caesarean birth on infant gut microbiota. Sci Rep. 2018; 8:13757. 